\title{
HYPER-STONEAN ENVELOPES OF COMPACT SPACES
}

\author{
H. GARTH DALES AND GRZEGORZ PLEBANEK
}

AbstraCt. Let $K$ be a compact space, and denote by $\widetilde{K}$ its hyperStonean envelope. We discuss the class of spaces $K$ with the property that $\widetilde{K}$ is homeomorphic to $\widetilde{\mathbb{I}}$, the hyper-Stonean envelope of the closed unit interval $\mathbb{I}$. Certainly each uncountable, compact, metrizable space $K$ belongs to this class. We describe several further classes of compact spaces $K$ for which $\widetilde{K}=\widetilde{\mathbb{I}}$. In fact, $\widetilde{K}=\widetilde{\mathbb{I}}$ if and only if the Banach spaces $M(K)$ and $M(\mathbb{I})$ of measures on $K$ and $\mathbb{I}$ are isometrically isomorphic.

\section{INTRODUCTION}

Let $K$ be a non-empty, locally compact (Hausdorff) space. Then $C_{0}(K)$ denotes the commutative $C^{*}$-algebra of all complex-valued, continuous functions on $K$ that vanish at infinity; in particular, $C_{0}(K)$ is a Banach algebra. For every Banach algebra $A$, the bidual space $A^{\prime \prime}$ has two products, denoted by $\square$ and $\diamond$, with respect to which $A^{\prime \prime}$ is a Banach algebra and such that the natural embedding of $A$ into $A^{\prime \prime}$ identifies $A$ as a closed subalgebra of $\left(A^{\prime \prime}, \square\right)$ and $\left(A^{\prime \prime}, \diamond\right)$; these two products are called the first and second Arens products on $A^{\prime \prime}$, following [1]. The Banach algebra $A$ is Arens regular if the two products $\square$ and $\diamond$ coincide on $A^{\prime \prime}$; when $A$ is commutative, this occurs if and only if $\left(A^{\prime \prime}, \square\right)$ is also commutative. For the basic theory of these products, see $[4, \S 2.6]$ or $[5, \S 3.2]$.

In fact, each $C^{*}$-algebra $A$ is Arens regular [4, Theorem 3.2.36]; indeed the bidual $\left(A^{\prime \prime}, \square\right)$ is a von Neumann algebra, called the enveloping von Neumann algebra of $A$. Thus the bidual $C_{0}(K)^{\prime \prime}$ of a commutative $C^{*}$ algebra $C_{0}(K)$ is itself a commutative, unital $C^{*}$-algebra, and so, by the Gel'fand-Naimark theorem, has the form $C(\widetilde{K})$ for a compact space $\widetilde{K}$; this space $\widetilde{K}$ is the hyper-Stonean envelope of $K$ [5, Definition 5.4.2]. This approach gives an abstract realization of the space $\widetilde{K}$.

2010 Mathematics Subject Classification. Primary 46H10 46J10, 46B25; Secondary 28A33, $28 \mathrm{C} 15$.

Key words and phrases. Hyper-Stonean envelope, Borel measure, Maharam type, biduals of Banach algebras, Arens products, measure separable spaces, Eberlein compact, Rosenthal compact, Corson compact.

The second author was supported by the Polish National Science Center research grant NCN 2013/11/B/ST1/03596 (2014-2017). 
For example, the hyper-Stonean envelope of the space $\mathbb{N}$ is the StoneCech compactification $\beta \mathbb{N}$ of $\mathbb{N}$. On the other hand, let us take $K$ to be the closed unit interval $\mathbb{I}=[0,1]$. Then $\widetilde{\mathbb{I}}$ is a 'much bigger' space than $\beta \mathbb{N}$.

There are several ways of 'constructing' $\widetilde{K}$ given in the text [5]. A topological characterization of the space $\widetilde{\mathbb{I}}$ is given in [5, Theorem 6.5.4], and it is shown in [5, Theorem 6.5.6] that the cardinality of $\widetilde{\mathbb{I}}$ is $2^{2^{\mathrm{c}}}$; the cardinality of various other subsets of $\widetilde{\mathbb{I}}$ is determined in $[5, \S 6.6]$.

It is not hard to see that $\widetilde{\mathbb{I}}$ is equal to the space $\widetilde{K}$ for any uncountable, compact, metrizable space $K$; as we shall see, this follows from the fact that $M(K)$ and $M(\mathbb{I})$ are isometrically isomorphic as Banach spaces [5, Corollary 4.6.7]. However there are compact space $K$ such that $\widetilde{K}=\widetilde{\mathbb{I}}$, but such that $K$ is not metrizable, and so 'other spaces' than uncountable, compact, metrizable spaces have the same hyper-Stonean envelope as $\mathbb{I}$. One example of such a space is given in [5, Example 6.3.1]; it is the 'two-arrows space'.

The purpose of this note is to give a number of other examples of compact spaces $K$ such that $\widetilde{K}$ is homeomorphic to $\widetilde{\mathbb{I}}$.

\section{Measures}

The cardinality of a set $S$ is denoted by $|S|$; the cardinality of the continuum is $\mathfrak{c}$; the continuum hypothesis is termed ' $\mathrm{CH}$ ' and Martin's axiom is 'MA'.

The dual of a Banach space $E$ is denoted by $E^{\prime}$, and its bidual is $E^{\prime \prime}$. Let $E$ and $F$ be Banach spaces. The space of bounded linear operators from $E$ into $F$ is $\mathcal{B}(E, F)$. We write $E \sim F$ when $E$ and $F$ are isomorphic (and so there is a bijection in $\mathcal{B}(E, F))$ and $E \cong F$ when $E$ and $F$ are isometrically isomorphic (and so there is an isometric bijection in $\mathcal{B}(E, F)$ ).

Let $K$ be a non-empty, compact space. Then we denote by $M(K)$ the Banach space of all complex-valued, regular Borel measures on $K$, as in $[5, \S 4.1]$, so that $\|\mu\|=|\mu|(K)$ for $\mu \in M(K)$. Thus the dual Banach space of $C(K)$ is identified isometrically with $M(K)$, and $M(K)$ is an isometric predual of $C(\widetilde{K})$. Now suppose that $K$ and $L$ are compact spaces. Then $C(\widetilde{K})$ is isometrically isomorphic to $C(\widetilde{L})$ if and only if $\widetilde{K}$ and $\widetilde{L}$ are homeomorphic [5, Theorem 6.1.4], and so $\widetilde{K}$ and $\widetilde{L}$ are homeomorphic whenever $M(K) \cong M(L)$. On the other hand, in the case where, for a compact space $X$, the space $C(X)$ is isometrically a dual space (i.e., $C(X)$ is a von Neumann algebra), $C(X)$ has a unique isometric predual, identified with the space $N(X)$ of normal measures on $X$ [5, Theorem 6.4.2], and so $M(K) \cong M(L)$ when $\widetilde{K}$ and $\widetilde{L}$ are homeomorphic to each other. Thus two 
compact spaces $K$ and $L$ have the same hyper-Stonean envelope if and only if $M(K) \cong M(L)$.

Let $K$ be a non-empty, compact space. As in [5], we denote by $M_{d}(K)$ and $M_{c}(K)$, the closed linear subspaces of $M(K)$ consisting of the discrete and continuous measures, respectively, so that $M(K)=M_{d}(K) \oplus M_{c}(K)$; we write $P(K)$ for the set of probability measures in $M(K)$, and then set $P_{d}(K)=P(K) \cap M_{d}(K)$ and $P_{c}(K)=P(K) \cap M_{c}(K)$. Indeed, take a measure $\mu \in M(K)$. Then $\mu \in P_{c}(K)$ if and only if $\mu(K)=\|\mu\|=1$ and $\mu(\{x\})=0(x \in K)$. In fact, we have $P_{c}(K)=\{0\}$ if and only if the space $K$ is scattered, in the sense that each non-empty subset $A$ of $K$ contains a point that is isolated in $A$ [17]. Further, $P_{c}(K) \neq\{0\}$ if and only if there is a continuous surjection from $K$ onto $\mathbb{I}$ (see [25, 8.5.4 and 19.7.6]), in which case $\left|P_{c}(K)\right| \geq \mathfrak{c}$.

Lebesgue measure on $\mathbb{I}$ is denoted by $m$, so that $m \in P_{c}(\mathbb{I})$.

Suppose that $K$ and $L$ are compact spaces such that $C(K) \sim C(L)$. It seems to be unclear whether this always implies that $M(K) \cong M(L)$; see $\S 4$. It does follow that $|K|=|L|$ when $C(K) \sim C(L)$ [5, Corollary 6.1.6], and so $M_{d}(K) \cong M_{d}(L)$ in this case.

Let $K$ be a non-empty, compact space, and take $\mu \in P(K)$. As usual, $L^{1}(K, \mu)$ denotes the Banach space of all measurable functions on $K$ such that $\|f\|_{1}=\int_{K}|f| \mathrm{d} \mu<\infty$, where we identify equivalent functions $f$ and $g$ such that $\|f-g\|_{1}=0$. A measure $\mu \in P(K)$ has countable Maharam type if the Banach space $L^{1}(K, \mu)$ is separable; otherwise, $\mu$ has uncountable Maharam type. When $\mu$ is continuous and has countable Maharam type, von Neumann's isomorphism theorem [5, Corollary 4.4.13] shows that $L^{1}(K, \mu) \cong L^{1}(\mathbb{I}, m)$. In general, the Maharam type of a measure $\mu \in P(K)$ is defined as the density of the Banach space $L^{1}(K, \mu)$; the measure is homogeneous if it has the same Maharam type when restricted to any subset of $K$ of positive measure. Let $\mu \in P_{c}(K)$ be a homogeneous measure of type $\kappa$. Then, by the Maharam theorem, $L^{1}(K, \mu)$ is isometrically isomorphic to $L^{1}\left(\mathbb{I}^{\kappa}, m_{\kappa}\right)$, where $\kappa$ is an infinite cardinal and $m_{\kappa}$ is the usual product measure on the Tikhonov cube $\mathbb{I}^{\kappa}$; see Lacey [16, §14] and Fremlin [8, p. 531].

For a non-empty set $T$, we denote by $\ell^{1}(T)$ the Banach space of all functions on $T$ such that $\sum_{t \in T}|f(t)|<\infty$, with the usual $\ell^{1}$-norm.

The following definition of measure separable spaces was given by Lacey [16, p. 175]; see also Džamonja and Kunen [6] for a clear account and some examples. 
Definition 2.1. The class of non-empty, compact spaces $K$ such that each $\mu \in P(K)$ has countable Maharam type is denoted by (MS).

It is not difficult to check that the class (MS) contains all metrizable and all scattered compact spaces, and that this class is closed under continuous images and countable products. We shall give more examples of spaces from the class (MS) below.

Let $K$ be a non-empty, compact space. A family $\mathcal{F}$ of measures in $P(K)$ is singular if $\mu \perp \nu$ whenever $\mu, \nu \in \mathcal{F}$ and $\mu \neq \nu$ [5, Definition 4.6.1]. Each singular family of measures is contained in a maximal (with respect to inclusion) singular family of measures [5, §4.6]. For example, it is shown in [5, Corollary 5.2.8] that, for each uncountable, compact, metrizable space $K$, every maximal singular family in $P(K)$ that contains all the point masses consists of $\mathfrak{c}$ point masses and $\mathfrak{c}$ continuous measures, and this implies that $|P(K)|=\left|P_{c}(K)\right|=\mathfrak{c}$.

We shall use some standard remarks about Banach lattices.

Let $E$ and $F$ be Banach lattices. An operator $T \in \mathcal{B}(E, F)$ is disjointnesspreserving if $|T x| \perp|T y|$ in $F$ whenever $|x| \perp|y|$ in $F$. Now take an operator $T \in \mathcal{B}(E, F)$ that is order-bounded and disjointness-preserving. By [20, Theorem 3.1.4], the modulus $|T|$ of $T$ exists, and $|T|: E \rightarrow F$ is a Banachlattice homomorphism. In the case where $T$ is an isometric isomorphism, $|T|$ is also an isometric isomorphism. When $E$ and $F$ are $A L$-spaces, every bounded operator from $E$ to $F$ is regular, and hence order-bounded.

Let $K$ be a compact space. Then $C(K)$ and $M(K)$ are (complex) Banach lattices in the usual way; see [5] and [20], for example. Indeed, $M(K)$ is an $A L$-space. By Kakutani's representation theorem for $A L$-spaces (see [20, Theorem 2.7.1] or [25, §26.3], for example),

$$
M(K)=\bigoplus\left\{L^{1}(K, \mu): \mu \in \mathcal{F}\right\}
$$

where $\mathcal{F}$ is a maximal singular family in $P(K)$ and the sum is an $\ell^{1}$-sum; $c f$. also $[12,13,16]$. Such a Kakutani decomposition yields Theorem 2.3, below. However we first give a (known) proposition.

Proposition 2.2. Let $K$ and $L$ be non-empty, compact spaces, and suppose that $M(K) \cong M(L)$. Then there is an isometric Banach-lattice isomorphism from $M(K)$ onto $M(L)$.

Proof. Note that $M(K)$ and $M(L)$ are $A L$-spaces as Banach lattices and that every linear isometry from $M(K)$ onto $M(L)$ is disjointness-preserving [5, Corollary 4.2.6], and so this follows from the above remarks. 
The above proposition also follows from Maharam's theorem; see Theorem 4.2 , below.

Theorem 2.3. Let $K$ be a non-empty, compact space. Then the following are equivalent:

(a) $M(K) \cong M(\mathbb{I})$;

(b) there is an isometric Banach-lattice isomorphism from $M(K)$ onto $M(\mathbb{I})$;

(c) $\widetilde{K}=\widetilde{\mathbb{I}}$;

(d) $M(K) \sim M(\mathbb{I})$;

(e) $M(K)$ is isometric to the $\ell^{1}$-sum of $\ell^{1}(\mathbb{I})$ and of $\mathfrak{c}$-many copies of $L^{1}(\mathbb{I}, m)$;

(f) $K$ is in the class (MS) and $|P(K)|=\left|P_{c}(K)\right|=\mathfrak{c}$.

Proof. The implications (b) $\Rightarrow(\mathrm{a}) \Rightarrow(\mathrm{d})$ are trivial, and (a) $\Rightarrow$ (b) by Proposition 2.2; the equivalence of (a) and (c) has been noted above; the implication (f) $\Rightarrow$ (e) above is essentially proved by Lacey [16]; see Theorem 5 on page 175 . The implication $(\mathrm{a}) \Rightarrow(\mathrm{e})$ follows by using a maximal singular family in $P(K)$ of the form mentioned above, and (f) $\Rightarrow$ (a) follows directly from Kakutani's decomposition theorem and the fact that $L^{1}(K, \mu) \cong L^{1}(\mathbb{I}, m)$ whenever $\mu \in P_{c}(K)$ has countable Maharam type.

The implication $(d) \Rightarrow$ (a) was noted by Rosenthal in [24], Remark on page 244. We shall give a separate proof of this implication in Corollary 4.4, below.

To prove the remainding implication, that (e) $\Rightarrow$ (f), of Theorem 2.3, we first recall the following fact (see, e.g., [14, Lemma 7.2(ii)]).

Lemma 2.4. Let $\left\{X_{\gamma}: \gamma \in \Gamma\right\}$ be a family of Banach spaces, and let $X=\bigoplus_{\gamma \in \Gamma} X_{\gamma}$ be its $\ell^{1}$-sum. For each weakly compact set $L \subset X$ and each $\varepsilon>0$, there is a finite set $I \subset \Gamma$ such that

$$
\sum_{\gamma \in \Gamma \backslash I}\left\|x_{\gamma}\right\|<\varepsilon \quad\left(x=\left(x_{\gamma}\right)_{\gamma} \in L\right) .
$$

Lemma 2.5. Let $\kappa$ be an infinite cardinal. Then the space $L^{1}\left(\mathbb{I}^{\kappa}, m_{\kappa}\right)$ does not embed isomorphically into an $\ell^{1}$-sum of spaces of density $<\kappa$.

Proof. Recall that the subset $B$ of $L^{1}\left(\mathbb{I}^{\kappa}, m_{\kappa}\right)$ consisting of the functions $g$ with $\|g\|_{\infty} \leq 1$ is weakly compact. Let $\pi_{\xi}: \mathbb{I}^{\kappa} \rightarrow \mathbb{I}$ be the projection onto the 
$\xi$ th coordinate, regarded as an element of $L^{1}\left(\mathbb{I}^{\kappa}, m_{\kappa}\right)$. Then, by elementary calculations, $\left\|\pi_{\xi}-\pi_{\eta}\right\|_{1}=1 / 3$ whenever $\xi \neq \eta$.

Suppose that $T: L^{1}\left(\mathbb{I}^{\kappa}, m_{\kappa}\right) \rightarrow \bigoplus_{\gamma \in \Gamma} X_{\gamma}$ is an isomorphic embedding, where each $X_{\gamma}$ is a Banach space. Then the image $T[B]$ is weakly compact, and so there exists $c>0$ such that $\left\|T\left(\pi_{\xi}\right)-T\left(\pi_{\eta}\right)\right\| \geq c$ for every $\xi \neq \eta$. Applying Lemma 2.4 with $\varepsilon=c / 2$, we obtain a finite set $I \subset \Gamma$ such that the norm of the projection of $T\left(\pi_{\xi}\right)-T\left(\pi_{\eta}\right)$ onto $\bigoplus_{\gamma \in I} X_{\gamma}$ is at least $c / 2$ whenever $\xi \neq \eta$. We conclude that the density of $X_{\gamma}$ is at least $\kappa$ for some $\gamma \in I$, and this gives the result.

\section{Proof. (of Theorem 2.3.)}

$(\mathrm{e}) \Rightarrow(\mathrm{f})$ Since $K$ satisfies (e), the space $K$ belongs to (MS) by Lemma 2.5. Moreover (e) clearly implies that $|P(K)|=\left|P_{c}(K)\right|=\mathfrak{c}$.

Let $K$ be an uncountable, compact, metrizable space. Then $K$ satisfies clause (e) of the above theorem, and hence it satisfies all the other clauses; cf. [4, Corollary 4.6.7].

We note that it is shown in [9] that, under some mild set-theoretic axioms, there is a compact space $K$ and a maximal singular family in $P(K)$ of cardinality strictly greater than $|K|$. In particular, under Martin's axiom, MA, one can construct such a space $K$ with $|K|=\mathfrak{c}$ and such that there is a maximal singular family in $P(K)$ of cardinality $2^{\mathfrak{c}}$. However, it is not clear whether there is a compact space $K$ in the class (MS) such that $|K|=\mathfrak{c}$ and $|P(K)|>\mathfrak{c}$, and so it is conceivable that the conditions in Theorem 2.3 are also equivalent to the condition that $K$ be a non-scattered space in the class (MS) and $|K|=\mathfrak{c}$.

We now give some further examples of compact spaces in the class (MS).

(1) Let $K$ be a compact space, and take $\mu \in P(K)$. Then $\mu$ is countablydetermined if there is a countable family $\mathcal{F}$ of closed subsets of $K$ such that

$$
\mu(U)=\sup \{\mu(F): F \subset U, F \in \mathcal{F}\}
$$

for each non-empty, open subset $U$ of $K$. It is clear that a countablydetermined measure is of countable Maharam type.

Compact spaces $K$ such that each $\mu \in K$ is countably-determined form the class $\mathcal{C}$ discussed by Mercourakis in [19]. The class $\mathcal{C}$ is contained in the class (MS); it includes all scattered compact spaces, all totally ordered compact spaces, and all Eberlein compact spaces (a compact space is Eberlein if it is homeomorphic to a subset of a 
Banach space with the weak topology). The class $\mathcal{C}$ is closed under countable products, continuous images, and closed subspaces.

It is easy to check that there is a separable, closed subspace $L$ of $K$ such that $\mu(L)=1$ whenever $\mu \in P(K)$ is countably-determined. In fact, every countably-determined $\mu \in P(K)$ admits a $\mu$-uniformly distributed sequence $\left(x_{n}\right)$, that is, a sequence whose means converge to $\mu$ in the weak-* topology of $P(K)$, in the sense that

$$
\lim _{n \rightarrow \infty} \frac{1}{n} \sum_{i=1}^{n} f\left(x_{i}\right)=\int_{K} f \mathrm{~d} \mu \quad(f \in C(K)) ;
$$

see [19, Corollary 2.8].

There are consistent examples of compact spaces that are in (MS), but that are outside the class $\mathcal{C}$; see Example 3.5 below, and see [3] for a further discussion. It seems to be an open question whether such an example can be constructed in the usual set theory.

(2) A compact space is Rosenthal compact if it is homeomorphic to a subspace of $B_{1}(X)$, the space of functions of the first Baire class (see [5, Definition 3.3.6]) on a complete, separable, metric space $X$. It follows that $|K| \leq \mathfrak{c}$ for every Rosenthal compact space $K$.

Every compact, metrizable space is Rosenthal compact, but there are separable, non-metrizable Rosenthal compact spaces such as the 'two-arrows' space, and there are non-separable examples. It follows from the very definition that no space of cardinality $>\mathfrak{c}$ is Rosenthal compact, so there are Eberlein compacta that are not Rosenthal compact. On the other hand, the 'two-arrows' space is not Eberlein compact since it is separable, but not metrizable.

Suppose that $K$ and $L$ are compact spaces such that $K$ is Rosenthal compact and $C(K) \sim C(L)$. Then $L$ is also Rosenthal compact [10, Proposition 11].

It is shown in [26] that each Rosenthal compact space belongs to the class (MS); some history of this result is given in [18]. It is an open problem whether every Rosenthal compact space is in the class $\mathcal{C}$; see [18] for a partial positive solution.

(3) Let $K$ be a compact space, and consider $P(K)$ equipped with the relative weak-* topology, $\sigma(M(K), C(K))$. It is an open problem whether $K$ must be in (MS) whenever $P(K)$ has countable tightness. Recall that a topological space $X$ has countable tightness if, for every $A \subset X$ and $x \in \bar{A}$, there is a countable set $B \subset A$ such that $x \in \bar{B}$. This problem and its connections with other properties of 
$C(K)$ spaces are discussed in [23], where the following is proved: if $P(K \times K)$ has countable tightness, then $K$ is in (MS). This result is a generalization of the fact that the class (MS) contains all Rosenthal compacta.

(4) A compact space is Corson compact if it is homeomorphic to a subspace of the space

$$
\left\{x=\left(x_{\alpha}\right) \in \mathbb{R}^{\kappa}:\left|\left\{\alpha<\kappa: x_{\alpha} \neq 0\right\}\right| \leq \omega\right\}
$$

for some cardinal $\kappa$. Every Eberlein compact space is Corson compact. It is not difficult to check from the very definition that every separable Corson compact is metrizable. Hence every separable, non-metrizable Rosenthal compact space is not Corson compact. It is shown in [2] that, under $\mathrm{MA}+\neg \mathrm{CH}$, all Corson compact spaces belong to the class (MS). There are examples constructed under $\mathrm{CH}$ (or weaker axioms) of Corson compact spaces that are not in (MS); see Example 3.5, below, and Haydon [12].

(5) It is shown by Fremlin in [7] that, in the theory $\mathrm{ZFC}+\mathrm{MA}+\neg \mathrm{CH}$, all compact spaces that cannot be mapped onto $\mathbb{I}^{\omega_{1}}$ are in the class (MS). Since no Corson compact space can be mapped onto $\mathbb{I}^{\omega_{1}}$, Fremlin's result is not provable within the usual set theory. Fremlin's theorem is related to the so-called Haydon problem that originates in [11]; see [22] for a short survey.

\section{Hyper-Stonean ENVELOPES}

We shall now use Theorem 2.3 to show that the hyper-Stonean envelopes $\widetilde{K}$ of various compact space $K$ are equal to $\widetilde{\mathbb{I}}$.

Theorem 3.1. Let $K$ be a non-empty, Rosenthal compact space of cardinality $\mathfrak{c}$.

(i) Suppose that $K$ is scattered. Then $\widetilde{K}=\beta \mathbb{I}_{d}$, the Stone-Cech compactification of a discrete space of cardinality $\mathfrak{c}$.

(ii) Suppose that $K$ is not scattered. Then $\widetilde{K}=\widetilde{\mathbb{I}}$.

Proof. By remark (2), above, the space $K$ belongs to the class (MS).

(i) Since $K$ is scattered, every $\mu \in P(K)$ is purely atomic, and so $M(K)$ is isometric to $\ell^{1}(K) \cong \ell^{1}\left(\mathbb{I}_{d}\right)$. Hence $C(K)^{\prime \prime}$ is isometrically isomorphic to $\ell^{1}\left(\mathbb{I}_{d}\right)^{\prime} \cong \ell^{\infty}\left(\mathbb{I}_{d}\right) \cong C\left(\beta \mathbb{I}_{d}\right)$.

(ii) Since $K$ is not scattered, there is a continuous surjection from $K$ onto $\mathbb{I}$ and $\left|P_{c}(K)\right| \geq \mathfrak{c}$. A theorem of Godefroy [10, Proposition 7] shows 
that $P(K)$ is also a Rosenthal compact space with respect to the relative weak-* topology, and so $|P(K)| \leq \mathfrak{c}$. Thus $|P(K)|=\left|P_{c}(K)\right|=\mathfrak{c}$, and so the result follows from Theorem 2.3 , (f) $\Rightarrow$ (c).

Theorem 3.2. Let $K$ be a non-empty, compact space in the class $\mathcal{C}$ such that $K$ is not scattered and $|K|=\mathfrak{c}$. Then $\widetilde{K}=\widetilde{\mathbb{I}}$.

Proof. We again have $\left|P_{c}(K)\right| \geq \mathfrak{c}$ since $K$ is not scattered. On the other hand, by Mercourakis' theorem mentioned above, every $P(K)$ has a $\mu^{-}$ uniformly distributed sequence. Since $|K|=\mathfrak{c}$, there are only $\mathfrak{c}$ sequences in $K$, and therefore $|P(K)| \leq \mathfrak{c}$. Thus $|P(K)|=\left|P_{c}(K)\right|=\mathfrak{c}$, and the result again follows from Theorem 2.3 .

Using the remarks in $\S 2$, Theorem 3.2 immediately yields the following consequences.

Corollary 3.3. Let $K$ be a non-empty, compact space such that $K$ is not scattered and $|K|=\mathfrak{c}$. Suppose further that:

(i) $K$ is a linearly ordered topological space; or

(ii) $K$ is Eberlein compact; or

(iii) $K$ is Corson compact and $\mathrm{MA}+\neg \mathrm{CH}$ holds.

Then $\widetilde{K}=\widetilde{\mathbb{I}}$.

Part (ii) of the above theorem was already effectively given by Rosenthal in his classic, foundational paper [24]; see [24, Proposition 5.5].

Theorem 3.4. Let $K$ be a non-empty, first-countable compact space that is not scattered. Then it is relatively consistent with $\mathrm{ZFC}$ that $\widetilde{K}=\widetilde{\mathbb{I}}$.

Proof. It is proved in [21] that it is relatively consistent with ZFC that each first-countable, compact space belongs to the class (MS) and also that $P(K)$ is a first-countable, compact space with respect to its weak-* topology. Since each first-countable space has cardinality at most $\mathfrak{c}$, it follows immediately that $|P(K)|=\left|P_{c}(K)\right|=\mathfrak{c}$, and so the result again follows from Theorem 2.3 .

Using the construction from [9], one can show that, under $\mathrm{CH}$, there is a first-countable compact space $K$ of cardinality $\mathfrak{c}$ such that $|P(K)|=2^{\mathfrak{c}}$, and so Theorem 3.4 is not provable in the usual set theory. 
Example 3.5. Assuming CH, Haydon [12] and Kunen [15] constructed two compact spaces $\mathbb{K}$ and $\mathbb{H}$ such that:

(i) $\mathbb{K}$ and $\mathbb{H}$ are first-countable Corson compact spaces of topological weight $\mathfrak{c}$;

(ii) $\mathbb{K}$ carries a strictly positive normal measure $\mu_{1}$ of countable Maharam type, and $\mathbb{H}$ carries a strictly positive normal measure $\mu_{2}$ of uncountable Maharam type;

(iii) if $K$ is either $\mathbb{K}$ or $\mathbb{H}$ and $\nu \in P(K)$ is such that $\nu$ is singular with respect to the corresponding measure $\mu_{1}$ or $\mu_{2}$, then $\nu$ has a metrizable support.

Recall that the main objective of Kunen's construction was to obtain under $\mathrm{CH}$ a compact $L$-space (that is, a space that is hereditarily Lindelöf, but non-separable), while the main purpose of Haydon's construction was to give a new type of a dual $L$-space. We should warn the reader that, to obtain the space $\mathbb{K}$ we mention here, one needs to use Kunen's original construction together with a remark on page 287 of [15].

Let us note that Kunen's space $\mathbb{K}$ is in the class (MS). Indeed, for each $\nu \in P(\mathbb{K})$, write $\nu=\nu^{\prime}+\nu^{\prime \prime}$, where $\nu^{\prime}$ is absolutely continuous with respect to $\mu_{1}$ and $\nu^{\prime \prime}$ is its singular part. Then clearly $\nu^{\prime}$ has countable Maharam type, and this is also true of the measure $\nu^{\prime \prime}$ because it is concentrated on a metrizable subspace. For the same reason, we conclude that $|P(\mathbb{K})|=\left|P_{c}(\mathbb{K})\right|=\mathfrak{c}$, and hence that $M(\mathbb{K}) \cong M(\mathbb{I})$ and $\widetilde{\mathbb{K}}=\widetilde{\mathbb{I}}$. Note also that the measure $\mu_{1}$ is not countably-determined since $\mu_{1}$ does not have a separable support (recall that a separable Corson compact space is necessarily metrizable).

For Haydon's space $\mathbb{H}$, we have $|P(\mathbb{H})|=\left|P_{c}(\mathbb{H})\right|=\mathfrak{c}$ for the same reason; however, $\mathbb{H}$ is not in the class (MS), and so it follows from Theorem 2.3, (a) $\Rightarrow(\mathrm{f})$, that $M(\mathbb{H}) \approx M(\mathbb{I})$.

\section{ISOMETRIC AND ISOMORPHiC TYPES OF $M(K)$ SPACES}

In this section, we shall consider when the fact that two spaces of the form $M(K)$ are isomorphic implies that they are isometrically isomorphic (and hence that there is an isometric Banach-lattice isomorphism between them). More explicitly, we raise the following problems.

Problem 4.1. Let $K$ and $L$ be two non-empty, compact spaces.

(i) Is it true that $M(K) \cong M(L)$ whenever $M(K) \sim M(L)$ and also $|K|=|L|$ ? 
(ii) Does $C(K) \sim C(L)$ imply that $M(K) \cong M(L)$ ?

(iii) Do either of questions (i) or (ii) have a positive answer under the additional assumption that $|K|=|L|=\mathfrak{c}$ ?

Let us recall that $C(K) \sim C(L)$ implies that $|K|=|L|$, see [5, Corollary 6.1.6]. We shall mention below an example showing that the assumption that $M(K) \sim M(L)$ alone need not imply that $|K|=|L|$; see Example 4.7.

In this section, we shall try to analyze question (iii) of Problem 4.1; it is naturally related to the problem that we are studying. We follow here some considerations presented by Haydon in [13].

Consider two Banach spaces each of which can be expressed as an $\ell^{1}$ direct sum of spaces of the form $L^{1}(\mu)$. Haydon [13, page 22] gave an example of a pair of such spaces that are mutually isomorphic, but are not isometrically isomorphic. However Haydon's spaces are not of the form $M(K)$.

Let $K$ be a non-empty, compact space. When we wish to decompose $M(K)$ as an $\ell^{1}$-direct sum of spaces of the form $L^{1}(\mu)$, we can do so by using a maximal family $\mathcal{F}$ in $P(K)$ of mutually singular homogeneous measures. Note, in particular, that $\mu, \nu \in P(K)$ are mutually singular whenever they are homogeneous and have different Maharam types. It follows that $M(K)$ is isometrically isomorphic to an $\ell^{1}$-direct sum of $\ell^{1}\left(K_{d}\right)$ and spaces of the form $L^{1}\left(\mathbb{I}^{\kappa}, m_{\kappa}\right.$ ) (here $\kappa$ is an infinite cardinal number), where each summand appears $\tau_{\kappa}(K)$ many times. In other words, we define $\tau_{\kappa}(K)$ as the (minimum) cardinality of a maximal family in $P(K)$ of mutually singular homogeneous measures of type $\kappa$. To have $\tau_{\kappa}(K)$ well-defined, we suppose that $\tau_{\kappa}(K)$ is either 0 (no measures of type $\kappa$ ) or 1 , or else $\tau_{\kappa}(K)$ is uncountable. Indeed, a countable family of mutually singular measures can be replaced by a single measure.

We note that $\tau_{\eta}(K)>0$ for every $\eta<\kappa$ whenever $\tau_{\kappa}(K)>0$; see, e.g., $[8,531 \mathrm{E}(\mathrm{f})]$. It is not difficult to check that a compact space $K$ cannot carry a measure of type exceeding the topological weight $w(K)$ of $K$. Since $w(K) \leq|K|$, we have $\tau_{\kappa}(K)=0$ whenever $\kappa>|K|$. Moreover, always $|P(K)| \leq 2^{w(K)}$, and so, in particular, $\tau_{\kappa}(K) \leq 2^{|K|}$ for every cardinal number $\kappa$.

The following theorem is essentially implicitly mentioned by Haydon on page 22 of [13].

Theorem 4.2. Let $K$ and $L$ be two non-empty, compact spaces. Then the following are equivalent: 
(a) there is an isometric Banach-lattice isomorphism between $M(K)$ and $M(L)$;

(b) the Banach spaces $M(K)$ and $M(L)$ are isometrically isomorphic;

(c) $|K|=|L|$ and $\tau_{\kappa}(K)=\tau_{\kappa}(L)$ for every infinite cardinal number $\kappa$.

Proof. The implication (a) $\Rightarrow(\mathrm{b})$ is obvious, and the implication (c) $\Rightarrow$ (a) follows from the fact that (c) implies that $M(K)$ and $M(L)$ have literally the same Kakutani decompositions.

To prove that $(\mathrm{b}) \Rightarrow(\mathrm{c})$, consider a linear isometry $T$ from $M(K)$ onto $M(L)$. Then $T\left(P_{c}(K)\right)=P_{c}(L)$ and $T\left(P_{d}(K)\right)=P_{d}(L)$ (by [5, Corollary 4.2.8]), and so $|K|=|L|$.

Recall also that $T \mu \perp T \nu$ when $\mu, \nu \in M(K)$ and $\mu \perp \nu$ because $\mu \perp \nu$ is equivalent to the metric condition $\|\mu+\nu\|=\|\mu-\nu\|=\|\mu\|+\|\nu\|$; for this, see [5, Proposition 4.2.5].

Next note that, for $\mu, \nu \in M(K)$, the measure $\nu$ is absolutely continuous with respect to $\mu$, written $\nu \ll \mu$, if and only if:

for every $\nu_{1} \in M(K)$, we have $\nu_{1}=0$ whenever $\nu_{1} \perp \nu-\nu_{1}$ and $\nu_{1} \perp \mu$. Using this remark, we conclude that $\mu \ll \nu$ implies that $T \mu \ll T \mu$.

Now it follows that, for a fixed $\mu \in P_{c}(K)$, the operator $T$ maps $L^{1}(K, \mu)$ into $L^{1}(L, T \mu)$; hence the Maharam type of $T \mu$ is bigger than or equal to the type of $\mu$. Repeating this argument for $T^{-1}$, we conclude that $T$ preserves the Maharam type of continuous measures. Note that a measure $\mu \in P(K)$ is homogeneous of type $\kappa$ if and only if every $\nu \in P(K)$ satisfying $\nu \ll \mu$ has type $\kappa$; hence $T \mu$ is homogeneous whenever $\mu$ is homogeneous.

Finally, given an infinite cardinal number $\kappa$ and a maximal family

$$
\left\{\mu_{\xi}: \xi<\tau_{\kappa}(K)\right\}
$$

of mutually singular measures from $P(K)$, where every $\mu_{\xi}$ is homogeneous of type $\kappa$, then $\left\{T \mu_{\xi}: \xi<\tau_{\kappa}(K)\right\}$ witnesses the fact that $\tau_{\kappa}(L)=\tau_{\kappa}(K)$.

We shall now consider two non-empty, compact spaces $K$ and $L$ such that $M(K) \sim M(L)$.

Lemma 4.3. Let $K$ and $L$ be two non-empty, compact spaces such that $M(K) \sim M(L)$, and suppose that $K$ carries a homogeneous measure of Maharam type $\kappa$. Then $L$ also carries a homogeneous measure of Maharam type $\kappa$.

Proof. It follows from the assumptions that $L^{1}\left(\mathbb{I}^{\kappa}, m_{\kappa}\right)$ embeds isomorphically into $M(L)$. Hence, by Lemma $2.4, L$ carries a homogeneous measure of type $\geq \kappa$. By $[8,531 \mathrm{E}(\mathrm{f})]$, there is such a $\nu \in P(L)$ of type $\kappa$.. 
It is a consequence of Lemma 4.3 that the Kakutani decompositions of the two spaces $M(K)$ and $M(L)$ have the same summands whenever $M(K) \sim M(L)$ and $|K|=|L|$; it is unclear, however, whether these summands must appear with the same multiplicity, which would imply that $M(K) \cong M(L)$.

We have, however, the following particular case that gives a proof of the implication $(\mathrm{d}) \Rightarrow$ (a) of Theorem 2.3.

Corollary 4.4. For every compact space $K, M(K) \sim M(\mathbb{I})$ if and only if $M(K) \cong M(\mathbb{I})$.

Proof. Suppose that $M(K) \sim M(\mathbb{I})$. Then $\tau_{\kappa}(K)=0$ for every uncountable $\kappa$ by Lemma 4.3. Moreover, $\tau_{\omega}(K)=\mathfrak{c}$ since $K$ is not scattered. Finally, $|K|=\mathfrak{c}$ since $M(K)$ has density $\mathfrak{c}$. Thus $M(K) \cong M(\mathbb{I})$.

The above corollary gives the implication $(\mathrm{d}) \Rightarrow$ (a) of Theorem 2.3.

Assume now that $\mathfrak{c}=\omega_{1}$ and that $2^{\mathfrak{c}}=\omega_{2}$ (these assumptions are consequences of the generalized continuum hypothesis), and let us consider any compact space $K$ of cardinality $\mathfrak{c}$. Then the structure of the space $M(K)$ is fully determined by the values of the two cardinal numbers $\tau_{\omega}(K)$ and $\tau_{\omega_{1}}(K)$. The basic problem is:

Problem 4.5. Is it true that $\tau_{\omega_{1}}(K) \leq \tau_{\omega}(K)$ ?

Even if the answer to this problem is positive, we have quite a number of possibilities:

(1) $\tau_{\omega}(K)=0$, so that $\tau_{\omega_{1}}(K)=0$. Since $K$ is scattered, the space $M(K)$ is isometric to $\ell^{1}(K)$.

(2) $\tau_{\omega}(K)=\omega_{1}$ and $\tau_{\omega_{1}}(K)=0$. This case is described in Theorem 2.3.

(3) $\tau_{\omega}(K)=\omega_{1}$ and $\tau_{\omega_{1}}(K)=1$. This is the case for Haydon's space $\mathbb{H}$ that was mentioned in Example 3.5; the space $M(\mathbb{H})$ is isometrically isomorphic to the direct sum of $M(\mathbb{I})$ and $L^{1}\left(\mathbb{I}^{\omega_{1}}, m_{\omega_{1}}\right)$.

(4) $\tau_{\omega}(K)=\tau_{\omega_{1}}(K)=\omega_{1}$. This is the case for $K$ being the one-point compactification of $\omega_{1}$ disjoint copies of $\mathbb{H}$, for example.

(5) $\tau_{\omega}(K)=\tau_{\omega_{1}}(K)=\omega_{2}$. This is the case for the space $K$ given in Theorem 4.6 below.

(6) $\tau_{\omega}(K)=\omega_{2}$ and $\tau_{\omega_{1}}(K) \in\left\{0,1, \omega_{1}\right\}$. We do not know any examples of spaces $K$ that satisfy any of these three possibilities. 
The following theorem follows immediately from a result given in [9] and its modification that is presented in the appendix, below.

Theorem 4.6. Under $\mathrm{CH}$, there is a compact space $K$ of cardinality $\mathfrak{c}$ such that $\tau_{\omega}(K)=\tau_{\omega_{1}}(K)=2^{\mathfrak{c}}$.

Proof. By [9, Theorem 3A], there is a compact space $K_{1}$ of cardinality $\mathfrak{c}$ with $\tau_{\omega}\left(K_{1}\right)=2^{\mathfrak{c}}$. Theorem A.3, below, gives a compact space $K_{2}$ of cardinality $\mathfrak{c}$ with $\tau_{\omega_{1}}\left(K_{2}\right)=2^{\mathfrak{c}}$. Take $K$ to be the topological disjoint union of $K_{1}$ and $K_{2}$. Then $K$ has the required properties.

Example 4.7. Under $\mathrm{CH}$, there are two compact sets $K$ and $L$ such that $M(K) \sim M(L)$, but such that $M(K) \nRightarrow M(L)$.

Proof. Let $K$ be the space from Theorem 4.6. Take a discrete set $X$ of cardinality $2^{\mathfrak{c}}$ and its one-point compactification $\alpha X=X \cup\left\{x_{0}\right\}$, where $x_{0} \in K$. Then $L=K \cup X$ is a compact space.

We see that $M(K) \neq M(L)$ simply because $|K| \neq|L|$.

On the other hand, $M(K) \sim M(L)$, which can be demonstrated as follows. Note that $M(K)$ and $M(L)$ can be written as $\ell^{1}$-sums:

$$
\begin{aligned}
& M(K)=\ell^{1}(K) \oplus \bigoplus_{\xi<2^{\mathfrak{c}}} L^{1}(\mathbb{I}, m) \oplus Z ; \\
& M(L)=\ell^{1}(L) \oplus \bigoplus_{\xi<2^{\mathfrak{c}}} L^{1}(\mathbb{I}, m) \oplus Z ;
\end{aligned}
$$

because every continuous measure on $L$ is concentrated on $K$ (here $Z$ represents all the remaining summands).

It is therefore not difficult to check that $M(K)$ may be seen as a complemented subspace of $M(L)$ and that $M(L)$ is isomorphic to a complemented subspace of $M(K)$. Hence $M(K) \sim M(L)$ by the Pełczyński decomposition method described in [5, Theorem 2.4.9], in [13], 2A and the example on page 22 , and in many other places.

Finally, let us suppose that Problem 4.5 has a negative solution and that $\mathrm{CH}$ holds: take a compact space $L$ of cardinality $\mathfrak{c}$ such that $\tau_{\omega}(L)=\omega_{1}$ and $\tau_{\omega_{1}}(L)=\omega_{2}$, and take a space $K$ as in Theorem 4.6, so that $\tau_{\omega}(K)=\omega_{2}$ and $\tau_{\omega_{1}}(K)=\omega_{2}$. Then $M(K) \sim M(L)$; this may be checked by an argument analogous to that of Example 4.7.

On the other hand, it follows from Theorem 4.2 that $M(K)$ is not isometrically isomorphic to $M(L)$ for these two spaces $K$ and $L$, Thus our special hypothesis would give a counter-example to Problem 4.1, clause (i). 


\section{Appendix A. A COMpaCt SpaCE With A LARGE FAMily of MEASURES}

Theorem A.3, given below, is a modification of the main result from [9]. We shall use here the classical Stone duality between Boolean algebras and compact zero-dimensional spaces. Given a Boolean algebra $\mathfrak{A}$, its Stone space (consisting of all the ultrafilters on $\mathfrak{A}$ ) is denoted by $\operatorname{St}(\mathfrak{A})$. Suppose that $K$ is a compact, zero-dimensional space. Then $\operatorname{clop}(K)$ denotes the Boolean algebra of clopen subsets of $K$. Recall that the Stone duality is defined by

$$
\mathfrak{A} \ni a \rightarrow \widehat{a} \in \operatorname{clop}(S t(\mathfrak{A})), \quad \widehat{a}=\{\mathcal{F} \in S t(\mathfrak{A}): a \in \mathcal{F}\}
$$

see [5, Theorem 1.7.2], for example.

A family $\mathcal{G}$ is said to generate a Boolean algebra $\mathfrak{A}$ whenever $\mathfrak{A}$ is the smallest Boolean subalgebra of $\mathfrak{A}$ containing $\mathcal{G}$. Recall also that the family $\mathcal{G}$ is centred if $\bigwedge \mathcal{G}_{0} \neq 0$ for any finite subset $\mathcal{G}_{0}$ of $\mathcal{G}$.

Lemma A.1. Suppose that a Boolean algebra $\mathfrak{A}$ is generated by a family $\mathcal{G} \subset \mathfrak{A}$ which contains no uncountable, centred subfamily. Then the space $\operatorname{St}(\mathfrak{A})$ is Corson compact and $|K| \leq|\mathcal{G}|^{\omega}$.

Proof. Define a map $f: S t(\mathfrak{A}) \rightarrow 2^{\mathcal{G}}$, where $f(\mathcal{F})(G)=1$ if $G \in \mathcal{F}$ and $f(\mathcal{F})(G)=0$ otherwise. Then $f$ is continuous. Moreover, $f$ is injective since $\mathcal{G}$ generates $\mathfrak{A}$. Since every ultrafilter on $\mathfrak{A}$ contains at most countably many generators from $\mathcal{G}$, the set $f[S t(\mathfrak{A})]$ is contained in the space $\Sigma\left(2^{\mathcal{G}}\right)$, consisting of elements of $2^{\mathcal{G}}$ with countable support. Clearly, the cardinality of $\Sigma\left(2^{\mathcal{G}}\right)$ is bounded by $|\mathcal{G}|^{\omega}$.

Remarks A.2. (i) Let $\mu$ be a finitely-additive probability measure on a Boolean algebra $\mathfrak{A}$. Then $\mu$ can be transferred to the set function $\widehat{\mu}$ on $\operatorname{clop}(S t(\mathfrak{A}))$ by the formula $\widehat{\mu}(\widehat{a})=\mu(a)(a \in \mathfrak{A})$. In turn, $\widehat{\mu}$ extends uniquely to a Radon measure on $S t(\mathfrak{A})$ (which will still be denoted by $\widehat{\mu})$.

(ii) Suppose, further, that $\mathcal{G} \subset \mathfrak{A}$ is such an uncountable family and that $\mu(a \triangle b) \geq \varepsilon$ for some $\varepsilon>0$ whenever $a, b \in \mathcal{G}$ are distinct. Then the Maharam type of $\widehat{\mu}$ is uncountable.

(iii) Suppose that $\nu$ is another finitely-additive probability measure on $\mathfrak{A}$ and that, for every $\varepsilon>0$, there is $a \in \mathfrak{A}$ such that $\mu(a)<\varepsilon$ and $\nu(a)>1-\varepsilon$. Then $\widehat{\mu}$ and $\widehat{\nu}$ are mutually singular on $S t(\mathfrak{A})$. 
Theorem A.3. Assume that II can be covered by $\omega_{1}$ Lebesgue-null sets. Then there is a compact space $K$ of cardinality $\mathfrak{c}$ such that $K$ carries $2^{\omega_{1}}-$ many mutually singular Radon measures of uncountable type.

Proof. Take a family $\left\{N_{\xi}: \xi<\omega_{1}\right\}$ such that $m\left(N_{\xi}\right)=0$ for every $\xi<\omega_{1}$ and $\bigcup_{\xi<\omega_{1}} N_{\xi}=\mathbb{I}$. We may suppose that $N_{\xi} \subset N_{\eta}$ whenever $\xi<\eta<\omega_{1}$.

We consider the Cantor cube $2^{\omega_{1}}=\{0,1\}^{\omega_{1}}$. For each $\xi<\omega_{1}$ and $i=0,1$, we set

$$
C_{\xi}^{i}=\left\{x \in 2^{\omega_{1}}: x_{\xi}=i\right\} .
$$

Given $\xi<\omega_{1}$, we choose closed sets $F_{\xi, k} \subset \mathbb{I} \backslash N_{\xi}$ with $\lim _{k} m\left(F_{\xi, k}\right)=1$, and we define a Boolean algebra $\mathfrak{A}$ of subsets of $\mathbb{I} \times 2^{\omega_{1}} \times 2^{\omega_{1}}$ to be the one generated by the family $\mathcal{G}$ of all the sets

$$
G_{\xi, k}^{i, j}=F_{\xi, k} \times C_{\xi}^{i} \times C_{\xi}^{j},
$$

where $\xi<\omega_{1}, k \in \mathbb{N}$, and $i, j \in\{0,1\}$. We shall check that $K=\operatorname{St}(\mathfrak{A})$ is our desired space.

Note that the family $\left\{F_{\xi, k}: \xi<\omega_{1}, k \in \mathbb{N}\right\}$ of closed sets contains no uncountable, centred family because it contains no uncountable subfamily with non-empty intersection. Indeed, for every $t \in \mathbb{I}$, there is $\xi_{0}$ such that $t \in N_{\xi}$ for every $\xi \geq \xi_{0}$ and, consequently, $t \notin F_{\xi, k}$ for each $\xi \geq \xi_{0}$ and $k \in \mathbb{N}$. It follows that $\mathcal{G}$ contains no uncountable, centred family either. Hence the space $K$ is Corson compact and $|K|=\omega_{1}^{\omega}=\mathfrak{c}$ by Lemma A.1.

Let $\lambda$ be the usual product measure on the space $2^{\omega_{1}}$. For $x \in 2^{\omega_{1}}$, we write $\delta_{x}$ for the corresponding Dirac measure. To every $x \in 2^{\omega_{1}}$ we associate the measure $\mu_{x}$ on $\mathfrak{A}$ that is the restriction of the product measure $m \otimes \delta_{x} \otimes \lambda$ to $\mathfrak{A}$.

Fix some $x \in 2^{\omega_{1}}$. For each $\xi<\eta<\omega_{1}$, taking $i=x_{\xi}$ and $j=x_{\eta}$, we see that

$$
G_{\xi, k}^{i, 0} \triangle G_{\eta, k}^{j, 0} \supset\left(F_{\xi, k} \cap F_{\eta, k}\right) \times\left(C_{\xi}^{i} \cap C_{\eta}^{j}\right) \times\left(C_{\xi}^{0} \triangle C_{\eta}^{0}\right),
$$

so that

$\mu_{x}\left(G_{\xi, k}^{i, 0} \triangle G_{\eta, k}^{j, 0}\right) \geq m\left(F_{\xi, k} \cap F_{\eta, k}\right) \cdot \lambda\left(C_{\xi}^{0} \triangle C_{\eta}^{0}\right)=m\left(F_{\xi, k} \cap F_{\eta, k}\right) \cdot(1 / 4) \rightarrow 1 / 4$

as $k \rightarrow \infty$. Using Remark A.2(ii), we conclude that $\widehat{\mu_{x}}$ has uncountable type.

Consider now $x, y \in 2^{\omega_{1}}$ with $x \neq y$, and choose $\xi<\omega_{1}$ with $x_{\xi} \neq y_{\xi}$. Suppose, for instance, that $x_{\xi}=0$ and $y_{\xi}=1$. Then

$$
\mu_{x}\left(G_{\xi, k}^{0, j}\right)=(1 / 2) \cdot m\left(F_{\xi, k}\right) \quad \text { for } \quad j=0,1,
$$


so that the set $A=G_{\xi, k}^{0,0} \cup G_{\xi, k}^{0,1}$ satisfies the conditions that $\mu_{x}(A)=m\left(F_{\xi, k}\right)$ while $\nu_{y}(A)=0$. Hence $\widehat{\mu_{x}}$ and $\widehat{\mu_{y}}$ are singular by Remark A.2(iii).

Let us note that essentially the same argument shows that there is a compact space $K$ of cardinality c carrying $2^{\kappa}$ mutually singular Radon measures of Maharam type $\kappa$ whenever $\kappa \leq \mathfrak{c}$ is a cardinal number such that $\mathbb{I}$ is a union of an increasing family $\left\{N_{\xi}: \xi<\kappa\right\}$ of Lebesgue null sets.

Acknowledgements. This note arose from conversations in Bȩdlewo during a conference on 'Transfinite methods in Banach spaces and algebras of operators', 17-23 July 2016. We are grateful to the organisers, A. Avilés, P. Koszmider, and N. J. Laustsen for the invitation to attend this conference and to Hanka and Wiesław Żelazko for generous hospitality.

We are also grateful to Frederick Dashiell for some valuable comments on a draft of this paper and to José Rodríguez for pointing out to us the short proof of Lemma 2.5 that is reproduced above.

\section{REFERENCES}

[1] R. Arens, The adjoint of a bilinear operation, Proc. American Math. Soc. 2 (1951), 839-848.

[2] S. Argyros, S. Mercourakis, and S. Negrepontis, Functional-analytic properties of Corson-compact spaces, Studia Math. 89 (1988), 197-229.

[3] P. Borodulin-Nadzieja and G. Plebanek, Measures on Suslinean spaces, Fund. Math. 235 (2016), 287-302.

[4] H. G. Dales, Banach Algebras and Automatic Continuity, London Math. Soc. Monographs, Volume 24, Clarendon Press, Oxford, 2000.

[5] H. G. Dales, F. K. Dashiell, Jr., A. T.-M. Lau, and D. Strauss, Banach Spaces of Continuous Functions as Dual Spaces, Canadian Mathematical Society Books in Mathematics, Springer, 2016.

[6] M. Džamonja and K. Kunen, Properties of the class of measure separable compact spaces, Fund. Math. 147 (1995), 261-277.

[7] D. H. Fremlin, On compact spaces carrying Radon measures of uncountable Maharam type, Fund. Math. 154 (1997), 295-304.

[8] D. H. Fremlin, Measure theory, Volume V, available online.

[9] D. H. Fremlin and G. Plebanek, Large families of mutually singular Radon measures, Bull. Polish Acad. Sci. Math. 51 (2003), 169-174.

[10] G. Godefroy, Compacts de Rosenthal, Pacific J. Math. 91 (1980), 293-306.

[11] R. Haydon, On Banach spaces which contain $l_{1}(\tau)$ and types of measures on compact spaces, Israel J. Math. 28 (1977), 313-327. 
[12] R. Haydon, On dual $L^{1}$-spaces and injective bidual Banach spaces, Israel J. Math. 31 (1978), 142-152.

[13] R. Haydon, Non-separable Banach spaces, in: Functional analysis: Surveys and recent results II K.-D. Bierstedt and B. Fuchssteiner (eds.), North-Holland, Amsterdam (1980), 19-30.

[14] M. Kačena, O. F. K. Kalenda, and J. Spurný, Quantitative Dunford-Pettis property, Adv. Math. 234 (2013), 488-527.

[15] K. Kunen, A compact L-space under CH, Topology Appl. 12 (1981), 283-287.

[16] H. E. Lacey, The Isometric Theory of Classical Banach Spaces, Die Grundlehren der mathematischen Wissenschaften, Band 208, Springer-Verlag, New York-Heidelberg, 1974 .

[17] A. T.-M. Lau and P. Mah, Normal structure in dual Banach spaces associated with a locally compact group, Trans. American Math. Soc. 310 (1988), 341-353.

[18] W. Marciszewski and G. Plebanek, On measures on Rosenthal compacta, J. Mathematical Analysis and Applications 385 (2012), 185-193.

[19] S. Mercourakis, Some remarks on countably determined measures and uniform distribution of sequences Monatshefte für Mathematik, 121 (1996), 79-111.

[20] P. Meyer-Nieberg, Banach Lattices, Universitex, Springer-Verlag, Berlin, 1991.

[21] G. Plebanek, Approximating Radon measures on first-countable compact spaces, Colloquium Math. 86 (2000), 15-23.

[22] G. Plebanek, On compact spaces carrying random measures of large Maharam type, Acta Univ. Carolinae Math. Phys. 43 (2002), 87-99.

[23] G. Plebanek and D. Sobota, Countable tightness in the spaces of regular probability measures, Fund. Math. 229 (2015), 159-169.

[24] H.P. Rosenthal, On injective Banach spaces and the spaces $L^{\infty}(\mu)$ for finite measure $\mu$, Acta Math. 124 (1970), 205-248.

[25] Z. Semadeni, Banach Spaces of Continuous Functions, Monografie Matematyczne, Instytut Matematyczny Polskiej Akademii Nauk, Volume 55, Warsaw, 1971.

[26] S. Todorčević, Compact subsets of the first Baire class, J. American Math. Soc. 12 (1999), 1179-1212.

Department of Mathematics and Statistics, University of Lancaster, LANCASTER LA1 4YF, United Kingdom

E-mail address: g.dales@lancaster.ac.uk

Institute of Mathematics, Wroclaw University, 50-Wroclaw, Poland

E-mail address: grzes@math.uni.wroc.pl 